\title{
Josephson effect in quasi one-dimensional unconventional superconductors
}

\author{
Yasuhiro Asano ${ }^{1}$ Yukio TANAKA $^{2}$, Yasunari TAnuma ${ }^{2}$, Kazuhiko KUROKI $^{4}$ and Hiroki Tsuchiura ${ }^{5}$ \\ ${ }^{1}$ Department of Applied Physics, Hokkaido University, Sapporo 060-8628, Japan. \\ 2 Department of Applied Physics, Nagoya University, Nagoya, 464-8603, Japan and Crest, Japan Science and Technology \\ Corporation (JST), Nagoya 464-8063 Japan. \\ 3 Institute of Physics, Kanagawa University, Rokkakubashi, Yokohama, 221-8686, Japan. \\ ${ }^{4}$ Department of Applied Physics and Chemistry, The university of Electro-Communications, Chofu 182-8568, Japan. \\ 5 S.I.S.S.A. - Via Beirut 2-4 34014 Trieste, Italy.
}

(Received November 19, 2018)

\begin{abstract}
Josephson effect in junctions of quasi one-dimensional triangular lattice superconductors is discussed, where the theoretical model corresponds to organic superconductors (TMTSF) ${ }_{2} \mathrm{PF}_{6}$. We assume the quarter-filling electron band and $p, d$ and $f$ wave like pairing symmetries in organic superconductors. To realize the electronic structures in organic superconductors, we introduce the asymmetric hopping integral, $\left(t^{\prime}\right)$ among second nearest lattice sites. At $t^{\prime}=0$, the Josephson current in the $d$ wave symmetry saturates in low temperatures, whereas those in the $p$ and the $f$ wave symmetries show the low-temperature anomaly due to the zero-energy state at the junction interfaces. The low-temperature anomaly appears even in the $d$ wave symmetry in the presence of $t^{\prime}$, whereas the anomaly is suppressed in the $f$ wave symmetry. The shape of the Fermi surface is an important factor for the formation of the ZES in the quarter-filling electron systems.
\end{abstract}

KEYWORDS: organic superconductor, zero-energy states, low-temperature anomaly, quarter-filling

\section{Introduction}

Quasi one-dimensional (Q1D) superconductors $(\mathrm{TMTSF})_{2} \mathrm{X} \quad\left(\mathrm{X}=\mathrm{PF}_{6}, \quad \mathrm{ClO}_{4} \text {, etc. }\right)^{1,2)}$ have recently attracted much attention as a possible spin-triplet superconductor. A spin-triplet pairing has been suggested from an observation of large $H_{c 2}{ }^{3)}$ and unchanged Knight shift across $T_{c}{ }^{4)}$ Unconventional pairing with nodes on the Fermi surface has been suggested from an NMR measurement, ${ }^{5)}$ while a thermal conductivity measurement has reported the absence of nodes on the Fermi surface. ${ }^{6)}$ In addition, a recent experiment showed the zero-bias conductance peak (ZBCP) in tunnel spectra. ${ }^{7)}$ Theoretically, a $p$ wave paring in which nodes of the pair potential can be made to avoid intersecting the Q1D Fermi surface has been proposed in an early stage. ${ }^{8-10)}$ On the other hand, a spin-singlet $d$ wave pairing mediated by spin fluctuations has been proposed by several authors. ${ }^{11-13)}$ This is because superconductivity lies right next to the $2 k_{F}$ spin density wave (SDW) phase in the pressure-temperature phase diagram. Moreover, one of the present authors has recently proposed that ${ }^{14)}$ a triplet $f$ wave pairing may dominate over the $d$ and the $p$ wave in (TMTSF) $)_{2} \mathrm{PF}_{6}$ due to a combination of a Q1D Fermi surface, coexistence of the $2 k_{F}$ SDW and the $2 k_{F}$ charge density wave (CDW) suggested from a diffuse X-ray scattering, ${ }^{15,16)}$ and an anisotropy in the spin fluctuations. Thus the situation is not settled either experimentally or theoretically. In recent papers, we proposed the tunneling conductance experiment to determine which one of pairing symmetries is realized in (TMTSF $)_{2} \mathrm{X}^{17,18)}$ because the tunnel spectra are sensitive to the internal degree of freedom of Cooper pairs.

A great variety in the Andreev reflection ${ }^{19)}$ is one of interesting features in superconductors with unconventional pairing symmetries. The pair potential of a transmitted quasiparticle in the electron branch differs from that in the hole branch because of unconventional pairing symmetries. When the two pair potentials have opposite signs to each other, constructive interference effects of a quasiparticle near the surface of superconductors lead to the zero-energy state (ZES). ${ }^{20-25)}$ The ZES is observed as the ZBCP in tunneling conductance spectra. Since the high- $T_{c}$ superconductors have the $d$ wave pairing symmetry, ${ }^{26-31)}$ the ZBCP was found in a number of experiments. ${ }^{23,24,32-44)}$ So far a considerable number of theoretical studies have been made on the ZES itself ${ }^{20,21,25)}$ and related phenomena of transport properties in both spin-singlet ${ }^{45-61)}$ and spin-triplet ${ }^{62-68)}$ unconventional superconductor junctions. Effects of ferromagnets attaching to superconductors, ${ }^{69-76)}$ those of broken time-reversal symmetry states (BTRSS), ${ }^{77-89)}$ those of magnetic fields ${ }^{90-93)}$ and those of the random potentials ${ }^{94-105)}$ on the ZBCP are hot topics in recent studies. Since the ZES appears just on the Fermi energy, it drastically affects transport properties through the interface of unconventional superconductor junctions. The low-temperature anomaly of the Josephson current between the two unconventional superconductors is explained in terms of the resonant tunneling of Cooper pairs through the ZES. ${ }^{66,103,106-111)}$ The Josephson effect in high- $T_{c}$ superconductors has been reported in a number of papers. ${ }^{112-122)}$

In theories, the Andreev reflection in unconventional superconductors has been studied based on the free electron model in which the quadratic dispersion relation and the isotropic Fermi surface are assumed. Although the Fermi surface in real unconventional superconductors are not isotropic at al, the free electron model well ex- 
plains characteristic behaviors of the tunnel conductance and the Josephson current. This may be because such transport properties are sensitive to the pairing symmetries of superconductors. However recent studies show several exceptions. ${ }^{18,60)}$ For instance in high- $T_{c}$ superconductor junctions, the free electron model does not explain the Josephson current calculated on the twodimensional tight-binding model for some cases. ${ }^{60)}$ The low-temperature anomaly of the Josephson current is expected in the free electron model when the $a$ axis of the high- $T_{c}$ superconductors oriented from the junction interface normal. The anomaly is washed out by the Friedel oscillations of the wave function in the lattice simulation for specific orientation angles. In addition, the density of states at the surface of the superconductors is sensitive to the shape of the Fermi surface in Q1D organic superconductors (TMTSF $)_{2} \mathrm{X}$ when triangular lattice structures are taken into account. In such situation, the Josephson current is also expected to be sensitive to the pairing symmetries and the shape of the Fermi surface. In this paper, we discuss the direct-current Josephson effect motivated by the surface density of states in Q1D superconductors. ${ }^{17,18,123)}$ So far, a Josephson effect in organic superconductors has been reported in the theoretical paper. ${ }^{124)}$ The effects of electronic structures in QID lattice, however, are not taken into account.

This paper is organized as follows. In Sec. 2, we described the Josephson junctions of organic superconductors by the Bogoliubov-de Gennes equation on the twodimensional lattice. The Josephson current is discussed for $p, d$ and $f$ wave symmetries in Sec, 3. In Sec. 4, we discuss the calculated results. We summarize this paper in Sec. 5 .

\section{Model and method}

Let us consider Q1D superconductor / insulator / superconductor (SIS) junctions as shown in Fig. 1, where $\boldsymbol{r}=j \overline{\boldsymbol{x}}+m \overline{\boldsymbol{y}}$ labels a lattic site, where $\overline{\boldsymbol{x}}$ and $\overline{\boldsymbol{y}}$ are unit vectors in the $x$ and the $y$ directions, respectively. The two superconductors (i.e., $-\infty \leq j \leq 0$ and $L+1 \leq j \leq$ $\infty)$ are separated by the insulator (i.e., $1 \leq j \leq L$ ). We assume the periodic boundary condition in the $y$ direction and the number of lattice sites in the $y$ direction is $M$. The junctions are described by the mean-field Hamiltonian

$$
\begin{aligned}
& H_{\mathrm{BCS}}=\frac{1}{2} \sum_{\boldsymbol{r}, \boldsymbol{r}^{\prime}}\left[\tilde{c}_{\boldsymbol{r}}^{\dagger} h_{\boldsymbol{r}, \boldsymbol{r}^{\prime}} \hat{\sigma}_{0} \tilde{c}_{\boldsymbol{r}^{\prime}}-\tilde{c}_{\boldsymbol{r}}^{t} h_{\boldsymbol{r}, \boldsymbol{r}^{\prime}}^{*} \hat{\sigma}_{0}\left\{\tilde{c}_{\boldsymbol{r}^{\prime}}^{\dagger}\right\}^{t}\right] \\
& +\frac{1}{2} \sum_{\boldsymbol{r}, \boldsymbol{r}^{\prime} \in \mathrm{S}}\left[\tilde{c}_{\boldsymbol{r}}^{\dagger} \hat{\Delta}_{\boldsymbol{r}, \boldsymbol{r}^{\prime}}\left\{\tilde{c}_{\boldsymbol{r}^{\prime}}^{\dagger}\right\}^{t}-\left\{\tilde{c}_{\boldsymbol{r}}\right\}^{t} \hat{\Delta}_{\boldsymbol{r}, \boldsymbol{r}^{\prime}}^{*} \tilde{c}_{\boldsymbol{r}^{\prime}}\right], \\
& h_{\boldsymbol{r}, \boldsymbol{r}^{\prime}}=-t_{\boldsymbol{r}, \boldsymbol{r}^{\prime}}+\left(\epsilon_{\boldsymbol{r}}-\mu_{\boldsymbol{r}}\right) \delta_{\boldsymbol{r}, \boldsymbol{r}^{\prime}}, \\
& \hat{\Delta}_{\boldsymbol{r}, \boldsymbol{r}^{\prime}}=\left\{\begin{array}{ccc}
i \boldsymbol{d}_{\boldsymbol{r}, \boldsymbol{r}^{\prime}} \cdot \hat{\boldsymbol{\sigma}} \hat{\sigma}_{2}: \hat{r} & \text { triplet } \\
i d_{\boldsymbol{r}, \boldsymbol{r}^{\prime}} \hat{\sigma}_{2} & : & \text { singlet },
\end{array}\right. \\
& \tilde{c}_{\boldsymbol{r}}=\left(\begin{array}{c}
c_{\boldsymbol{r}, \uparrow} \\
c_{\boldsymbol{r}, \downarrow}
\end{array}\right)
\end{aligned}
$$

where $c_{\boldsymbol{r}, \sigma}^{\dagger}\left(c_{\boldsymbol{r}, \sigma}\right)$ is the creation (annihilation) operator of an electron at $\boldsymbol{r}$ with spin $\sigma=(\uparrow$ or $\downarrow), \hat{\sigma}_{0}$ is the $2 \times 2$ unit matrix representing the spin space, $\hat{\sigma}_{j}$ with
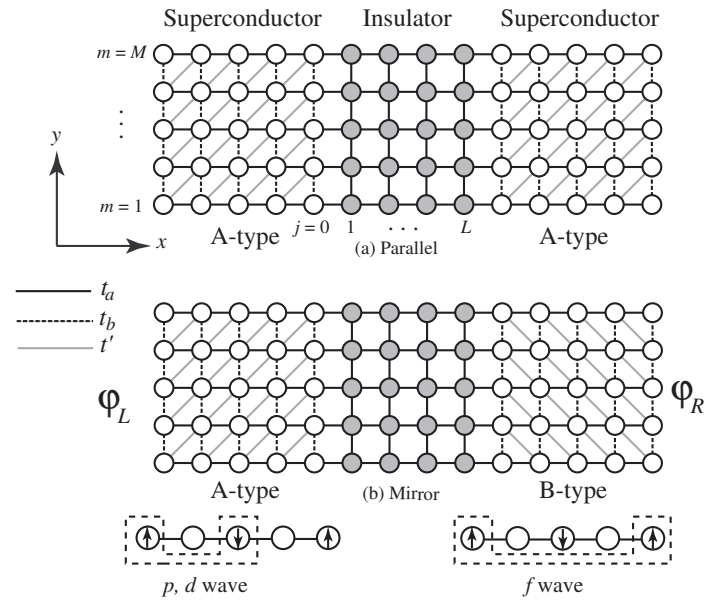

Fig. 1. A schematic figure of the SIS junction of the organic superconductor.

$j=1 \sim 3$ are the Pauli matrices and S in the summation denotes the superconductors. The Fermi energy in the superconductor is $\mu_{\boldsymbol{r}}=\mu_{S}$ for $j \leq 0$ and $j \geq L+1$, and that in the insulator is $\mu_{\boldsymbol{r}}=\mu_{N}$ for $1 \leq j \leq L$. In superconductors, we assume $\epsilon_{\boldsymbol{r}}=0$ and $t_{a}$ and $t_{b}$ are the hopping integral in the $x$ and $y$ directions, respectively. We also introduce asymmetric hopping integral $\left(t^{\prime}\right)$ between the second nearest neighbors as shown in Fig. 1 to realize electronic structures in (TMTSF) ${ }_{2} \mathrm{PF}_{6}$. In the insulator, $\epsilon_{\boldsymbol{r}}=V_{B}$ for $1 \leq j \leq L$ denotes the barrier potential and the hopping integrals in the two directions are equal to $t_{a}$. The pair potential in $p, d$ and $f$ wave symmetries are defined by,

$$
\begin{aligned}
& \boldsymbol{d}_{\boldsymbol{r}, \boldsymbol{r}^{\prime}}^{(p)}=\frac{\Delta}{2} e^{i \varphi_{i}} \operatorname{sgn}\left(j-j^{\prime}\right) \delta_{\left|j-j^{\prime}\right|, 2} \delta_{m, m^{\prime}} \boldsymbol{e}_{3}, \\
& d_{\boldsymbol{r}, \boldsymbol{r}^{\prime}}^{(d)}=\frac{\Delta}{2} e^{i \varphi_{i}} \delta_{\left|j-j^{\prime}\right|, 2} \delta_{m, m^{\prime}}, \\
& \boldsymbol{d}_{\boldsymbol{r}, \boldsymbol{r}^{\prime}}^{(f)}=\frac{\Delta}{2} e^{i \varphi_{i}} \operatorname{sgn}\left(j-j^{\prime}\right) \delta_{\left|j-j^{\prime}\right|, 4} \delta_{m, m^{\prime}} \boldsymbol{e}_{2},
\end{aligned}
$$

where $\varphi_{i}=\varphi_{L}$ or $\varphi_{R}$ is the macroscopic phase of superconductors and $\boldsymbol{e}_{j}$ with $j=1,2$ and 3 are unit vectors in the spin space. A schematic picture of Cooper pairs are shown in Fig. 1. The Hamiltonian is diagonalized by the Bogoliubov transformation,

$$
\begin{aligned}
{\left[\begin{array}{c}
\tilde{c}_{\boldsymbol{r}} \\
\left\{\tilde{c}_{\boldsymbol{r}}^{\dagger}\right\}^{t}
\end{array}\right] } & =\sum_{\lambda}\left[\begin{array}{cc}
\hat{u}_{\lambda}(\boldsymbol{r}) & \hat{v}_{\lambda}^{*}(\boldsymbol{r}) \\
\hat{v}_{\lambda}(\boldsymbol{r}) & \hat{u}_{\lambda}^{*}(\boldsymbol{r})
\end{array}\right]\left[\begin{array}{c}
\tilde{\gamma}_{\lambda} \\
\left\{\tilde{\gamma}_{\lambda}^{\dagger}\right\}^{t}
\end{array}\right], \\
\tilde{\gamma}_{\lambda} & =\left(\begin{array}{c}
\gamma_{\lambda, \uparrow} \\
\gamma_{\lambda, \downarrow}
\end{array}\right)
\end{aligned}
$$

where $\gamma_{\lambda, \sigma}^{\dagger}\left(\gamma_{\lambda, \sigma}\right)$ is creation (annihilation) operator of a Bogoliubov quasiparticle. In Eq. (8), $\hat{u}_{\lambda}$ and $\hat{v}_{\lambda}$ are the wavefunction of a quasiparticle which satisfy the Bogoliubov-de Gennes (BdG) equation. ${ }^{125)}$

$\sum_{\boldsymbol{r}^{\prime}}\left[\begin{array}{cc}h_{\boldsymbol{r}, \boldsymbol{r}^{\prime}} \hat{\sigma}_{0} & \hat{\Delta}_{\boldsymbol{r}, \boldsymbol{r}^{\prime}} \\ -\hat{\Delta}_{\boldsymbol{r}, \boldsymbol{r}^{\prime}}^{*} & -h_{\boldsymbol{r}, \boldsymbol{r}^{\prime}}^{*} \hat{\sigma}_{0}\end{array}\right]\left[\begin{array}{c}\hat{u}_{\lambda}\left(\boldsymbol{r}^{\prime}\right) \\ \hat{v}_{\lambda}\left(\boldsymbol{r}^{\prime}\right)\end{array}\right]=E_{\lambda}\left[\begin{array}{c}\hat{u}_{\lambda}(\boldsymbol{r}) \\ \hat{v}_{\lambda}(\boldsymbol{r})\end{array}\right]$. 
The eigenvalue $E_{\lambda}$ is independent of spin channels because we consider unitary states in superconductors. In what follows, we briefly discuss the method to calculate the Josephson current for the $d$ wave symmetry. The application to the $p$ and the $f$ wave symmetries is straightforward. In the case of the $d$ wave symmetry, the BdG equation in Eq. (10) is decoupled to two equations,

$$
\begin{aligned}
& \sum_{\boldsymbol{r}^{\prime}}\left[\begin{array}{cc}
h_{\boldsymbol{r}, \boldsymbol{r}^{\prime}} & d_{\boldsymbol{r}, \boldsymbol{r}^{\prime}}^{(d)} \\
\left(d_{\boldsymbol{r}, \boldsymbol{r}^{\prime}}^{(d)}\right)^{*} & -h_{\boldsymbol{r}, \boldsymbol{r}^{\prime}}^{*}
\end{array}\right]\left[\begin{array}{c}
\left(u_{11}\right)_{\lambda}\left(\boldsymbol{r}^{\prime}\right) \\
\left(v_{21}\right)_{\lambda}\left(\boldsymbol{r}^{\prime}\right)
\end{array}\right] \\
& =E_{\lambda}\left[\begin{array}{c}
\left(u_{11}\right)_{\lambda}(\boldsymbol{r}) \\
\left(v_{21}\right)_{\lambda}(\boldsymbol{r})
\end{array}\right],
\end{aligned}
$$

where $\left(u_{i j}\right)$, for example, represents an element of $\hat{u}$ in Eq. (8) and $\left[u_{21}, v_{11}\right]^{t}$ obeys essentially the same equation. In the following, we suppress $(1,1)$ of $\left(u_{11}\right)$ and $(2,1)$ of $\left(v_{21}\right)$. In this way, the wave function

$$
\Psi_{\lambda}(j)=\left(\begin{array}{c}
u_{\lambda}(j \overline{\boldsymbol{x}}+1 \overline{\boldsymbol{y}}) \\
\vdots \\
u_{\lambda}(j \overline{\boldsymbol{x}}+M \overline{\boldsymbol{y}}) \\
v_{\lambda}(j \overline{\boldsymbol{x}}+1 \overline{\boldsymbol{y}}) \\
\vdots \\
v_{\lambda}(j \overline{\boldsymbol{x}}+M \overline{\boldsymbol{y}})
\end{array}\right),
$$

satisfies the BdG equation. For $j<-2$, for instance, the BdG equation reads

$$
\begin{aligned}
& \frac{\Delta}{2}\left(\begin{array}{cc}
\hat{0} & e^{i \varphi_{L} \hat{1}} \\
e^{-i \varphi_{L}} \hat{1} & \hat{0}
\end{array}\right) \Psi_{\lambda}(j+2) \\
& +\left(\begin{array}{cc}
\hat{T}_{N}(-) & \hat{0} \\
\hat{0} & -\hat{T}_{N}(-)
\end{array}\right) \boldsymbol{\Psi}_{\lambda}(j+1) \\
& +\left(\begin{array}{cc}
-E_{\lambda} \hat{1}+\hat{E}_{S} & \hat{0} \\
\hat{0} & -E_{\lambda} \hat{1}-\hat{E}_{S}
\end{array}\right) \boldsymbol{\Psi}_{\lambda}(j) \\
& +\left(\begin{array}{cc}
\hat{T}_{N}(+) & \hat{0} \\
\hat{0} & -\hat{T}_{N}(+)
\end{array}\right) \boldsymbol{\Psi}_{\lambda}(j-1) \\
& +\frac{\Delta}{2}\left(\begin{array}{cc}
\hat{0} & e^{i \varphi_{L}} \hat{1} \\
e^{-i \varphi_{L} \hat{1}} & \hat{0}
\end{array}\right) \boldsymbol{\Psi}_{\lambda}(j-2)=0, \\
& \hat{T}_{N}(+)=\left(\begin{array}{ccccc}
-t_{a} & -t^{\prime} & 0 & \cdots & 0 \\
0 & -t_{a} & -t^{\prime} & \cdots & 0 \\
\vdots & \ddots & \ddots & \ddots & \vdots \\
0 & \cdots & 0 & -t_{a} & -t^{\prime} \\
-t^{\prime} & 0 & \cdots & 0 & -t_{a}
\end{array}\right) \\
& \hat{T}_{N}(-)=\left(\begin{array}{ccccc}
-t_{a} & 0 & \cdots & 0 & -t^{\prime} \\
-t^{\prime} & -t_{a} & 0 & \cdots & 0 \\
\vdots & \ddots & \ddots & \ddots & \vdots \\
0 & \cdots & -t^{\prime} & -t_{a} & 0 \\
0 & \cdots & 0 & -t^{\prime} & -t_{a}
\end{array}\right) \text {, } \\
& \hat{E}_{S}=\left(\begin{array}{ccccc}
-\mu_{S} & -t_{b} & 0 & \cdots & -t_{b} \\
-t_{b} & -\mu_{S} & -t_{b} & \cdots & 0 \\
\vdots & \ddots & \ddots & \ddots & \vdots \\
0 & \cdots & -t_{b} & -\mu_{S} & -t_{b} \\
-t_{b} & 0 & \cdots & -t_{b} & -\mu_{S}
\end{array}\right)
\end{aligned}
$$

where $\hat{1}$ and $\hat{0}$ are the $M \times M$ unit matrix and the zero matrix, respectively. To solve the BdG equation, we apply the recursive Green function method ${ }^{126-128)}$ and calculate the Matsubara Green function in a matrix form

$$
\check{G}_{\omega_{n}}\left(j, j^{\prime}\right)=\sum_{\lambda} \boldsymbol{\Psi}_{\lambda}(j)\left[i \omega_{n}-E_{\lambda}\right]^{-1} \boldsymbol{\Psi}_{\lambda}^{\dagger}\left(j^{\prime}\right),
$$

where $\omega_{n}=(2 n+1) \pi T$ is the Matsubara frequency and $T$ is a temperature. Throughout this paper, we use the units of $\hbar=k_{B}=1$, where $k_{B}$ is the Boltzmann constant. The Josephson current in the insulator $(1<j<L)$, is given by ${ }^{127-129)}$

$$
J(j)=-i e T \sum_{\omega_{n}} t_{a} \operatorname{Tr}\left[\check{G}_{\omega_{n}}(j+1, j)-\check{G}_{\omega_{n}}(j, j+1)\right] .
$$

We note that $J(j)$ is independent of $j$ when we consider the direct-current Josephson effect.

\section{Josephson current}

The low-temperature anomaly of the Josephson current is a typical phenomenon in the quantum transport between two unconventional superconductors. ${ }^{66,103,106-111)}$ In this section, we discuss effects of the asymmetric second nearest neighbor hopping $\left(t^{\prime}\right)$ on the ZES at the interface and on the Josephson current. For finite $t^{\prime}$, it is possible to consider two types of SIS junctions as shown in Fig. 1 (a) and (b). The parallel junction consists of two A-type superconductors, whereas the mirror-type junction consists of a A-type and a Btype superconductors. Generally speaking, the Josephson effect in the two junctions are not identical to each other.

In what follows, we choose parameters as $t_{b}=0.1 t_{a}$, $\mu_{S}=-1.4099 t_{a}, \mu_{N}=-2.0 t_{a}, M=20, L=4$, and $V_{B}=2.0 t_{a}$. The electron density is fixed at the quarterfilling. The amplitude of the pair potential at the zero temperature is $\Delta_{0}=0.1 t_{a}$ and the dependence of the pair potential on temperatures is described by the BCS theory.

\section{$3.1 p$ wave symmetry}

In Fig. 2, we show the Josephson current in the parallel junctions with the $p$ wave symmetry. The vertical axis is normalized by $\pi \Delta_{0} / 2 e R_{N}$, where $R_{N}$ is the normal resistance of junctions. We note in this vertical scale that the Josephson current of the $s$ wave symmetry with the isotropic Fermi surface is close to unity in the limit of the zero temperature. ${ }^{130)}$ In real materials, the second nearest neighbor hopping should be $-t^{\prime} / t_{a}=0.08$. We also show results for $-t^{\prime} / t_{a}=0$ and 0.16 for comparison. In Fig. 2 (a), the maximum value of the Josephson current $\left(J_{\max }\right)$ is plotted as a function of temperatures, where $\left(J_{\max }\right)$ is estimated from the Josephson current as a function of $\varphi=\varphi_{L}-\varphi_{R}$. The Josephson current increases with decreasing temperature irrespective of $t^{\prime}$ and does not saturate even in low temperatures. This behavior is called as the low-temperature anomaly of the Josephson current and is owing to the ZES forming at the junction interface. When the $\boldsymbol{d}$ vector has only one component in the free electron model, a condition for 

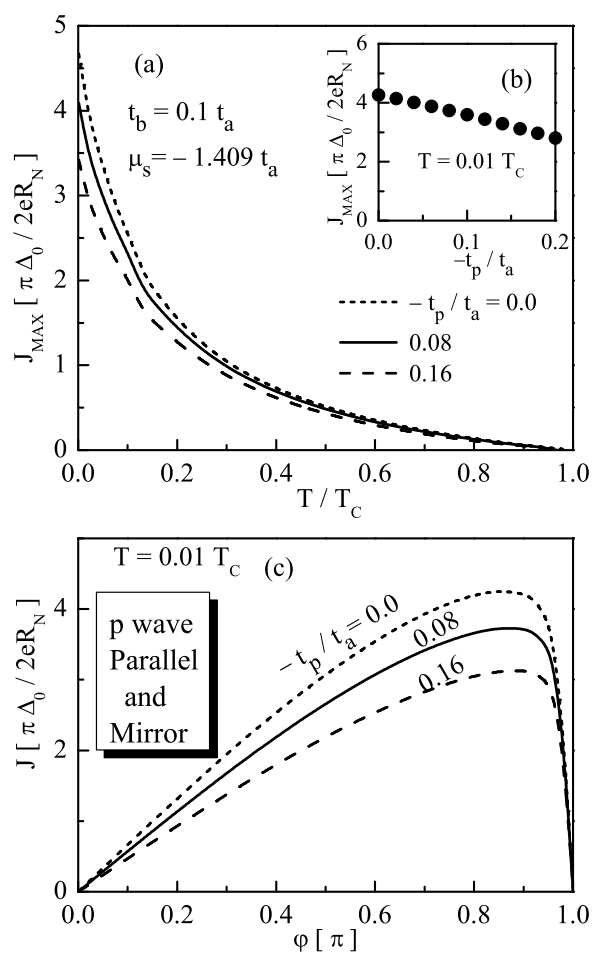

Fig. 2. Josephson current for the $p$ wave symmetry is shown, where $t_{b}=0.1 t_{a}, \mu_{S}=-1.4099 t_{a}, M=20, L=4$, and $V_{B}=2 t_{a}$. In (a), the maximum amplitude of the Josephson current $\left(J_{\max }\right)$ is plotted as a function of temperatures. In (b), $J_{\max }$ at $T=0.01 T_{c}$ are shown as a function of $-t^{\prime} / t_{a}$. The current-phase relation is calculated at $T=0.01 T_{c}$ in (c).

appearance of the ZES is given by

$$
\boldsymbol{d}\left(k_{a}, k_{b}\right) \cdot \boldsymbol{d}\left(-k_{a}, k_{b}\right)<0,
$$

where $\boldsymbol{d}\left(k_{a}, k_{b}\right)$ is the Fourier component of $\boldsymbol{d}_{\boldsymbol{r}-\boldsymbol{r}^{\prime}}$, and $k_{a}$ and $k_{b}$ are the wavenumber in the $x$ and $y$ directions, respectively. The two pair potentials in Eq. (19) corresponds to the pair potentials in the electron and the hole branch of a quasiparticle. Since the translational invariance in the $y$ holds, $k_{b}$ is conserved in the transmission and the reflection of a quasiparticle at the junction interfaces. In the $p$ wave symmetry, the pair potential in Eq. (5) results in

$$
\boldsymbol{d}^{(p)}\left(k_{a}, k_{b}\right)=\Delta \sin \left(2 k_{a}\right) \boldsymbol{e}_{3} .
$$

Since Eq. (20) is an odd function of $k_{a}$, it satisfies Eq. (19) for all the Fermi surface as shown in Fig. 3, where we draw the Fermi surface for $t^{\prime}=0$ in (a), $t^{\prime}=-0.08 t_{a}$ in the A-type superconductor in (b), and $t^{\prime}=-0.08 t_{a}$ in the B-type superconductor in (c) with the solid line. The pair potential in the $p$ wave symmetry has a node line at $k_{a}=0$ in both the free electron model and the lattice model. In the lattice model, however, Eq. (20) has additional node lines at $k_{a}= \pm 0.5 \pi$ because the pairing interaction works between two electrons on the second nearest neighbor sites at the quarter-filling as shown in Fig. 1. We define the additional node lines as the node lines appear in the pair potentials because
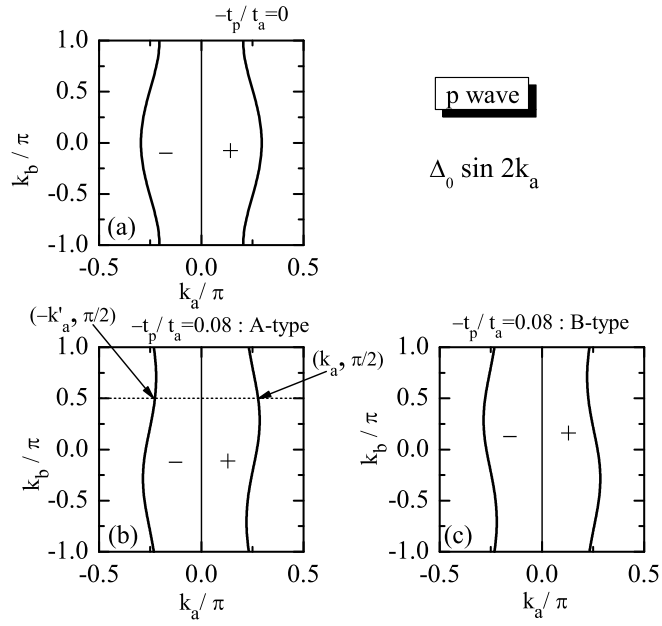

Fig. 3. The Fermi surface of TMTSF for $t^{\prime}=0$ is shown in (a). Those for $t^{\prime}=-0.08 t_{a}$ in the A-type and the B-type superconductors are shown in (b) and (c), respectively. The pair potential in the $p$ wave symmetry is given by $\Delta \sin 2 k_{a}$ which changes the sign at $k_{a}=0$ as indicated by + and - in the figures.

of the quarter-filled electron band on the Q1D lattice.

The asymmetric hopping $\left(t^{\prime}\right)$, modulates the shape of the Fermi surface as shown in Fig. 3 (b) and (c). When the Fermi surface looses a symmetry with respect to $k_{a}=$ 0 as in (b) and (c), the condition for the ZES should be rewrote as

$$
\boldsymbol{d}\left(k_{a}, k_{b}\right) \cdot \boldsymbol{d}\left(-k_{a}^{\prime}, k_{b}\right)<0
$$

where $k_{a}$ and $-k_{a}^{\prime}$ are the wave numbers of the Fermi surface for fixed $k_{b}$. In (b), the two wavenumbers are indicated by arrows on the Fermi surface for $k_{b}=\pi / 2$. In the following, we call Eq. (21) as the zero-energy condition (ZEC). Even in the presence of $t^{\prime}$, the ZEC is always satisfied because the additional node lines are far from the Fermi surface. The amplitude of the Josephson current at $T=0.01 T_{c}$ decreases with increasing $-t^{\prime} / t_{a}$ as show in Fig. 2 (b). This is mainly because $R_{N}$ decreases with increasing $-t^{\prime} / t_{a}$. In our calculation, $R_{N}$ in units of $h / e^{2}$ are 1.04, 0.83 and 0.63 for $-t^{\prime} / t_{a}=0,0.08$ and 0.16 , respectively. In Fig. 2 (c), the current-phase relation is calculated at $T=0.01 T_{c}$. The current-phase relation in a low temperature deviates from the sinusoidal function because the resonant tunneling through the ZES enhances the multiple Andreev reflection between the two superconductors. We note that the results in the mirrortype junction are identical to those in Fig. 2 (a)-(c). In the $p$ wave symmetry, the Josephson current are not qualitatively changed by introducing $t^{\prime}$ because the ZES at the interface governs the characteristic behavior of the Josephson current.

\section{2 d wave symmetry}

In Fig. 4, we show the Josephson current in the parallel junctions with the $d$ wave symmetry.

In (a), $J_{\max }$ is plotted as a function of temperatures. In the absence of $t^{\prime}$, the Josephson current saturates in 

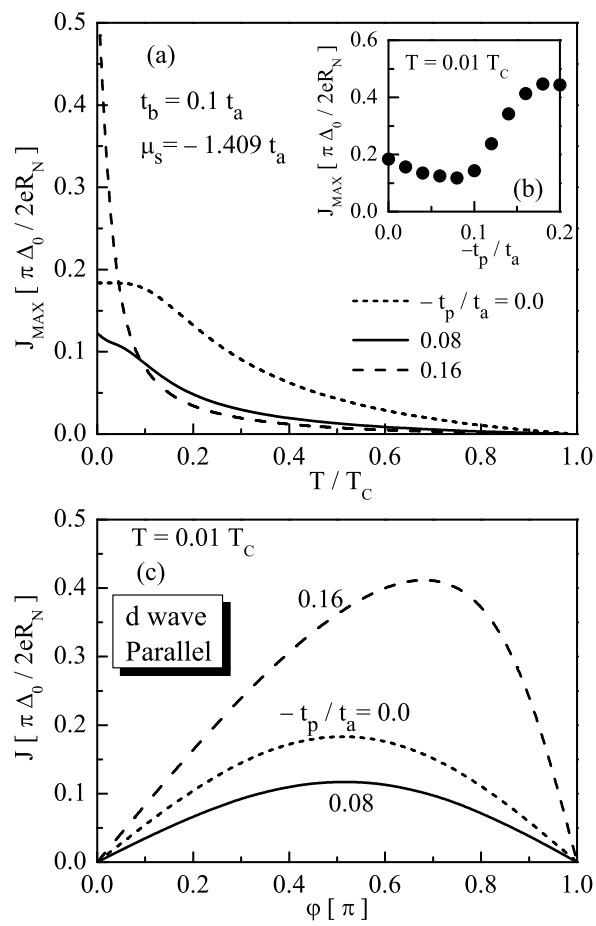

Fig. 4. Josephson current in the parallel junction with the $d$ wave symmetry is shown. In (a), the Josephson current is plotted as a function of temperatures. In (b), amplitudes of the Josephson current at $T=0.01 T_{c}$ are shown as a function of $-t^{\prime} / t_{a}$. The current-phase relation is calculated at $T=0.01 T_{c}$ in (c).
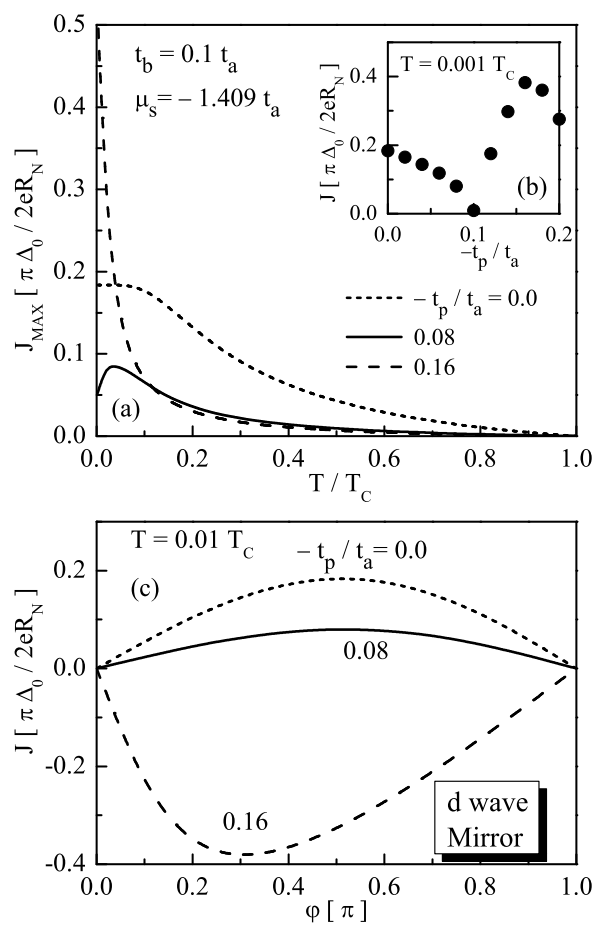

Fig. 5. Josephson current for the $d$ wave symmetry in the mirrortype junction.
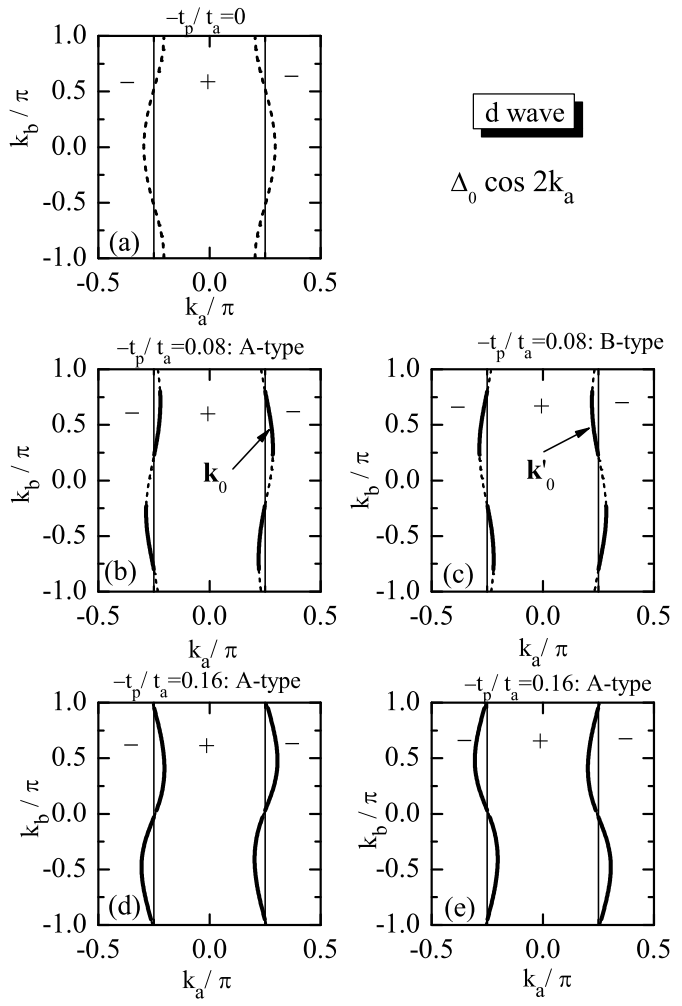

Fig. 6. The Fermi surface of TMTSF for $t^{\prime}=0$ is shown in (a). Those for $t^{\prime}=-0.08 t_{a}$ in the A-type and the B-type superconductors are shown in (b) and (c), respectively. The pair potential in the $d$ wave symmetry is given by $\Delta \cos 2 k_{a}$ which changes its sign at $k_{a}= \pm 0.25 \pi$ as indicated by + and - in the figures. The Fermi surface for $t^{\prime}=-0.16 t_{a}$ is shown for A-type and B-type superconductors in (d) and (e), respectively. The Fermi surface shown with the solid line satisfy the condition for the ZES in Eq. (22).

low temperatures as that in the $s$ wave junctions. On the other hand, the results for $-t^{\prime} / t_{a}=0.08$ and 0.16 do not saturate even in low temperatures. In particular, the Josephson current for $-t^{\prime} / t_{a}=0.16$ rapidly increases with decreasing temperatures. In the case of spin-singlet superconductors, a condition for appearance of the ZES is given by

$$
d\left(k_{a}, k_{b}\right) d\left(-k_{a}^{\prime}, k_{b}\right)<0,
$$

where $d\left(k_{a}, k_{b}\right)$ is the Fourier component of $d_{\boldsymbol{r}-\boldsymbol{r}^{\prime}}$. In the $d$ wave symmetry, the pair potential in Eq. (6) results in

$$
d^{(d)}\left(k_{a}, k_{b}\right)=\Delta \cos \left(2 k_{a}\right),
$$

and is the even function of $k_{a}$. The additional node lines in the lattice model are $k_{a}= \pm 0.25 \pi$ and $\pm 0.75 \pi$. In the absence of $t^{\prime}$, Eq. (23) does not satisfy the ZEC in Eq. (22) for all the Fermi surface as shown in Fig. 6, where we show the Fermi surface for $t^{\prime}=0$ in (a). The Fermi surface indicated by the broken line does not satisfy the ZEC. In the presence of $t^{\prime}$, however, the ZEC can be satisfied for some wave numbers on the Fermi surface as plotted with the solid line in (b) for $t^{\prime}=-0.08 t_{a}$. The sign of the pair potential changes frequently on the Fermi surface because $t^{\prime}$ modifies the shape of the Fermi sur- 
face lies along the additional node lines at $k_{a}= \pm 0.25 \pi$. As a result, the ZEC in Eq. (22) is satisfied for some wavenumbers, which leads to the formation of the ZES. This implies an importance of lattice structures on the quantum transport. The current-phase relation deviates from the sinusoidal relation as shown in the results for $-t^{\prime} / t_{a}=0.16$ in Fig. 4 (c) because most of the Fermi surface satisfy the ZEC as shown in Fig. 6 (d). We note that the Fermi surface around $k_{b}=0$ and $\pm \pi$ are still out of the ZEC for $-t^{\prime} / t_{a}=0.16$. The deviation for $-t^{\prime} / t_{a}=0.08$ from the sinusoidal relation is smaller than that for $-t^{\prime} / t_{a}=0.16$. A quasiparticle incident perpendicular to the junction interface mainly contributes to the Josephson current. The Fermi surface at $k_{b}=0$, however, does not satisfy the ZEC as shown in Fig. 6 (b). Thus the contribution of the resonant tunneling via the ZES is small for $-t^{\prime} / t_{a}=0.08$ even though the temperature dependence tends to be anomalous in low temperatures.

The Josephson effect in the mirror-type junctions is different from that in the parallel junctions because of the lattice structures. In Fig. 5, we show the Josephson current for the $d$ wave symmetry in the mirrortype junctions. In (a), $J_{\max }$ for $-t^{\prime} / t_{a}=0.08$ first increases with decreasing temperatures then decreases for $T<0.05 T_{c}$. Such non monotonic temperature dependence has been also reported in the high- $T_{c}$ superconductor Josephson junctions. For $-t^{\prime} / t_{a}=0.16$, the Josephson current changes its sign and the amplitude increases rapidly with decreasing temperatures. The differences between the parallel and the mirror-type junctions can be understood in terms of the relative sign of the pair potentials in the two superconductors. In the presence of the time-reversal symmetry, the Josephson current is decomposed into a series of

$$
J=\sum_{n=1}^{\infty} J_{n} \sin (n \varphi)
$$

It was shown that the $J_{1}$ is roughly given by ${ }^{111)}$

$$
J_{1}=\sum_{k_{b}} d_{R}\left(k_{a}^{\prime}, k_{b}\right) d_{L}\left(k_{a}, k_{b}\right) F_{1}\left(k_{b}\right),
$$

where $F_{1}$ is the positive function of $k_{b}, d_{R}\left(k_{a}^{\prime}, k_{b}\right)$ and $d_{L}\left(k_{a}, k_{b}\right)$ are the pair potential on the Fermi surface in the right and the left superconductors, respectively. Thus the product of $d_{R}\left(k_{a}^{\prime}, k_{b}\right) d_{L}\left(k_{a}, k_{b}\right)$ determines the sign of the Josephson current proportional to $\sin \varphi$. The parallel junction consists of two A-type superconductors as shown in Fig. 1 . Therefore $d_{R}\left(k_{a}, k_{b}\right)$ and $d_{L}\left(k_{a}, k_{b}\right)$ are identical to each other, which results in the positive sign of $J_{1}$. On the other hand in the mirror-type junctions, $d_{L}\left(\boldsymbol{k}_{0}\right)$ in the A-type superconductor shown in Fig. 6 (b) has the opposite sign to $d_{R}\left(\boldsymbol{k}_{0}^{\prime}\right)$ in the B-type superconductor in (c). It is easily shown that $d_{R}\left(k_{a}^{\prime}, k_{b}\right) d_{L}\left(k_{a}, k_{b}\right)$ is negative when the Fermi surface satisfies Eq. (22) in the mirror-type junctions. In high temperatures, a quasiparticle on the Fermi surface shown with the broken lines in Figs. 6 (b) and (c) dominates the Josephson current and $J_{1}$ is positive. This is because a quasiparticle incident perpendicular to the junction $\left(k_{b}=0\right)$ mainly contributes to the Josephson current and the contribution of the ZES is negligible. In low temperatures, the resonant transmission via the ZES also contributes to the Josephson current. As a consequence, $J_{1}$ has a non monotonic temperature dependence as shown in Fig. 5 (a) because the sign of the Josephson current via the ZES is negative. For $-t^{\prime} / t_{a}=0.16$, the Josephson current is negative for low temperatures because the resonant transmission via the ZES dominates the Josephson current. It is also shown that the Josephson current proportional to $\sin 2 \varphi$ is given by

$$
J_{2}=-\sum_{k_{b}}\left[d_{R}\left(k_{a}, k_{b}\right) d_{L}\left(k_{a}^{\prime}, k_{b}\right)\right]^{2} F_{2}\left(k_{b}\right),
$$

where $F_{2}$ is the positive function of $k_{b}$. The sign of $J_{2}$ is always negative irrespective of the pairing symmetries of the two superconductors. Thus the current-phase relation for $-t^{\prime} / t_{a}=0.16$ in Fig. 4 (c) takes its maximum at $\varphi>0.5 \pi$ in the parallel junctions. While that in the mirror-type junction takes its minimum at $\varphi<0.5 \pi$ in the mirror-junction in Fig. 5 (c).

\section{$3.3 f$ wave symmetry}

In Fig. 7, we show the Josephson current for the $f$ wave symmetry in the parallel junctions.

In (a), the maximum amplitude of the Josephson current is plotted as a function of temperatures for $-t^{\prime} / t_{a}$ $=0,0.08$ and 0.16. In the absence of $t^{\prime}$, the Josephson current show the low-temperature anomaly because the Fourier component of the pair potential is given by $\Delta \sin 4 k_{a}$ which satisfies Eq.(21) for all the Fermi surface as shown in Fig. 9 (a). The additional node lines are at $k_{a}= \pm 0.25 \pi, \pm 0.5 \pi$ and $\pm 0.75 \pi$ in the lattice model. The anomalous behavior tends to disappear for finite $t^{\prime}$. In Fig. 9 (b), the Fermi surface which do not satisfy Eq.(21) is indicated by the broken line for $t^{\prime}=-0.08 t_{a}$. For $t^{\prime}=-0.16 t_{a}$, most wave numbers of the Fermi surface do not satisfy Eq.(21) as shown in Fig. 9 (d). In contrast to the $d$ wave junctions, the ZES is suppressed by introducing $t^{\prime}$ in the $f$ wave junctions. The phasecurrent relation for $-t^{\prime} / t_{a}=0$ and 0.08 in Fig. 7 (c) apparently deviates from the sinusoidal function because of the multiple Andreev reflection via the ZES. The results for $-t^{\prime} / t_{a}=0.16$ slightly deviate from $\sin \varphi$, but degree of deviation is smaller than those for $-t^{\prime} / t_{a}=0$ and 0.08 .

In Fig. 8, we show the Josephson current for the $f$ wave symmetry in the mirror-type junctions. In (a), the Josephson current for $-t^{\prime} / t_{a}=0$ and 0.08 are essentially the same as those in the parallel junctions. In the currentphase relation for $-t^{\prime} / t_{a}=0.16$ in (c), the Josephson current becomes zero around $\varphi=0.75 \pi$. In this case, $J_{1}$ is negative because $\boldsymbol{d}_{R}\left(k_{a}^{\prime}, k_{b}\right) \cdot \boldsymbol{d}_{L}\left(k_{a}, k_{b}\right)$ is negative for almost all the Fermi surface. The amplitude of $J_{2}$ is not negligible because the resonant transmission through the ZES for a quasiparticle incident perpendicular to the interface $\left(k_{b}=0\right)$ enhances $J_{2}$. In a rough estimation, we find $\left|J_{2}\right| \sim 0.7\left|J_{1}\right|$.

\section{Discussion}

The formation of the ZES is a universal phenomenon at the surface of unconventional superconductors. ${ }^{25)}$ It may 

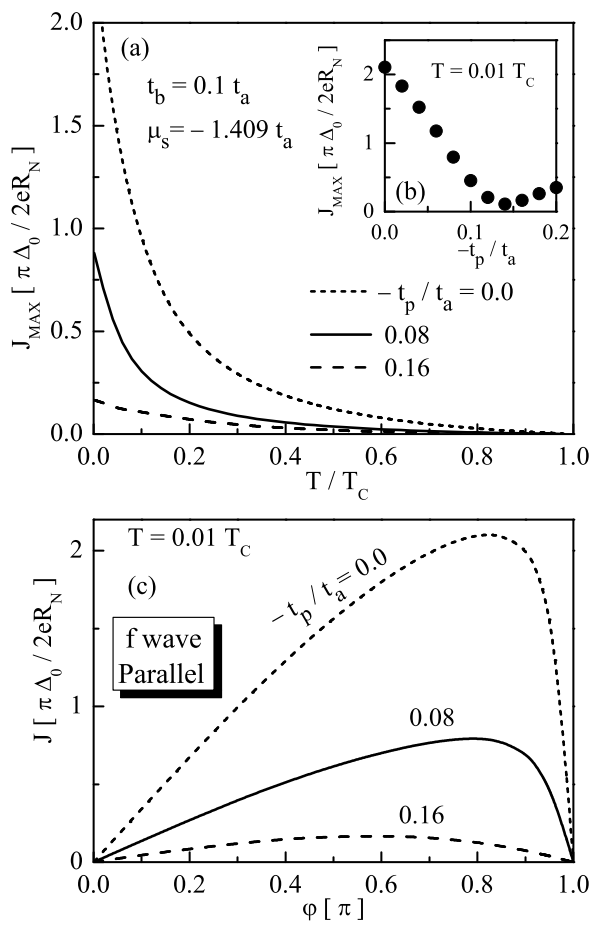

Fig. 7. Josephson current for $f$ wave symmetry in the parallel junctions. In (a), the maximum amplitude of the Josephson current is plotted as a function of temperatures. In (b), amplitudes of the Josephson current at $T=0.01 T_{c}$ are shown as a function of $-t^{\prime} / t_{a}$. The current-phase relation is calculated at $T=0.01 T_{c}$ in (c).
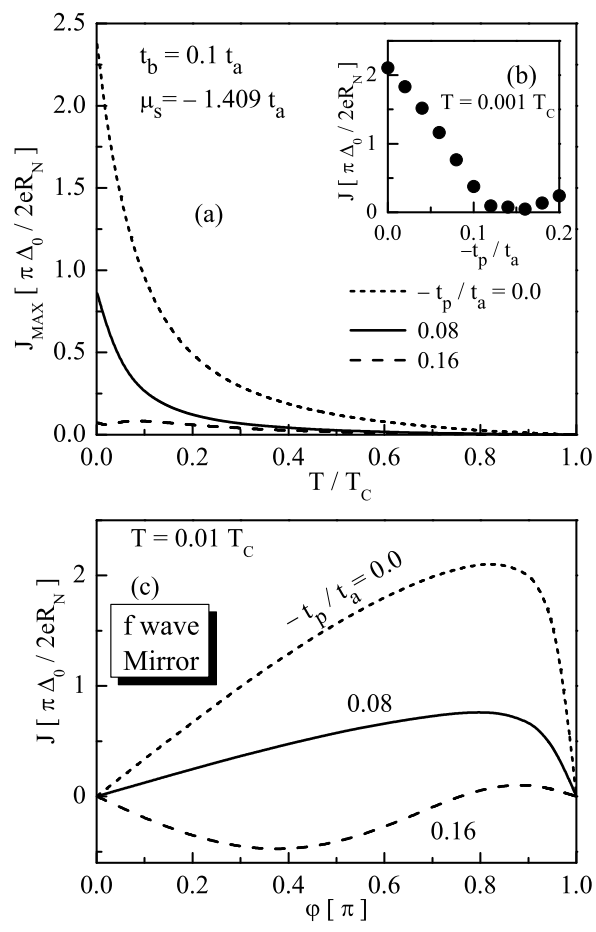

Fig. 8. Josephson current for $f$ wave symmetry in the mirror-type junctions.

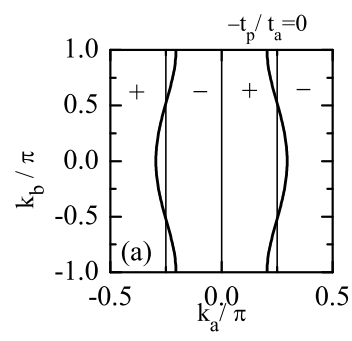

f wave

$\Delta_{0} \sin 4 \mathrm{k}_{\mathrm{a}}$
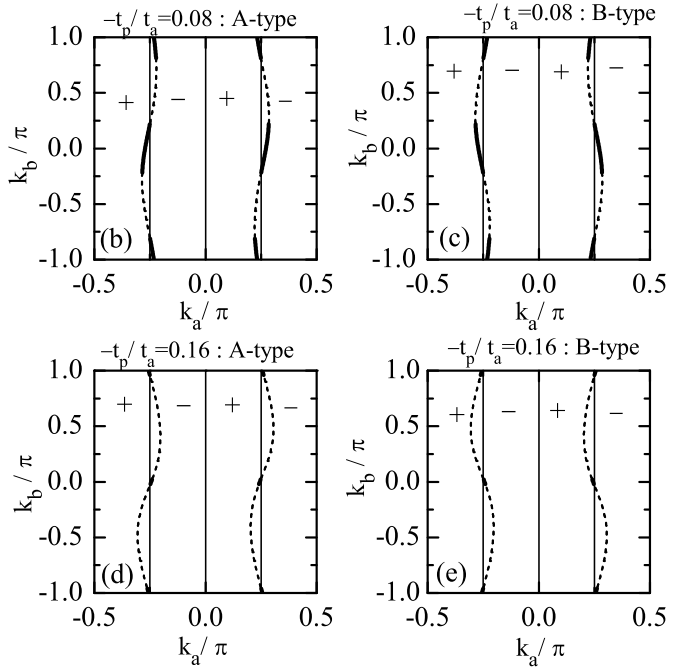

Fig. 9. The Fermi surface of TMTSF for $t^{\prime}=0$ is shown in (a). Those for $t^{\prime}=-0.08 t_{a}$ in the A-type and the B-type junctions are shown in (b) and (c), respectively. The Fermi surface for $t^{\prime}=-0.16 t_{a}$ is shown in (d) and (e). The pair potential in the $f$ wave symmetry is given by $\Delta \sin 4 k_{a}$ which changes the sign at $k_{a}=0$ and $\pm 0.25 \pi$ as indicated by + and - in the figures. The Fermi surface shown with the solid line satisfies the condition for the ZES. The broken line indicates the wave numbers of the Fermi surface which do not satisfy the condition of the ZES.

be possible to analyze the pairing symmetry of unconventional superconductors from the anisotropy in the tunneling conductance and that in the Josephson current. Actually we have investigated these transport properties in both the free electron model and the lattice model. As far as we study, there are no drastic differences between the two theoretical models in the case of high- $T_{c}$ superconductors. The formation of the ZES has been believed to be insensitive to the electronic structures such as the shape of the Fermi surface. The calculated results of this paper indicate that this statement is justified when the Fermi surface is far from the additional node lines peculiar to the lattice structures. We have shown that the shape of the Fermi surface strongly affects the formation of the ZES in Q1D organic superconductors. The Q1D quarter-filling band and the asymmetry of the Fermi surface with respect to $k_{a}$ are important factors for the ZES to be sensitive to the shape of the Fermi surface. In the quarter-filled band, the pairing interaction tends to work between two electrons at the second nearest neighbor sites in the $x$ direction as shown in Fig. 1 because of the long range Coulomb repulsion. In particular, the $f$ wave superconductivity requires the pairing correlation between the 4 th nearest neighbor sites in the $x$ direc- 
tion. As a consequence, the pair potential changes sign at additional node lines in the first Brillouin zone such as $k_{a}= \pm 0.5 \pi$ and $\pm 0.25 \pi$ as shown in Figs. 3, 6 and 9 . The asymmetric hopping $\left(t^{\prime}\right)$ which characterizes the triangular lattice structure causes the asymmetry of the Fermi surface with respect to $k_{a}$. The pair potential often changes its sign along the asymmetric Fermi surface because the Fermi surface in Q1D quarter-filled band lies along the additional node lines at $k_{a}= \pm 0.25 \pi$. In the $p$ wave symmetry, characteristic behaviors of the Josephson current are insensitive to $t^{\prime}$ because the Fermi surface is far from the additional node line at $k_{a}= \pm 0.5 \pi$. The sign change of the pair potential around the additional node lines at $k_{a}= \pm 0.25 \pi$ is very important for the formation of the ZES in the $d$ and $f$ wave symmetries. In the absence of $t^{\prime}$, it is clear that the ZES is formed in the $f$ wave symmetry and that the ZES is not formed in the $d$ wave symmetry. This is because the pair potential in the $f$ wave ( $d$ wave) symmetry is an odd (even) function of $k_{a}$. Roughly speaking, $t^{\prime}$ assists the formation of the ZES in the $d$ wave symmetry and suppresses the ZES in the $f$ wave symmetry. As a results, the Josephson current exhibit the various temperature dependences and the phase current relations depending on the pairing symmetries, the degree of asymmetry and the types of junctions.

\section{Conclusion}

We have studied the Josephson current in quasi onedimensional unconventional superconductors with triangular lattice structures. The theoretical model describes the organic superconductors such as (TMTSF) $)_{2} \mathrm{X}$. In the calculation, we assume $p, d$, and $f$ wave like pairing symmetries in superconductors. The pair potentials have the additional node lines because the pairing interaction works between the second or 4th nearest neighbor sites in the current direction at the quarter-filled electron band. The triangular lattice structure is characterized by the asymmetric second nearest neighbor hopping $\left(t^{\prime}\right)$ introduced on the square tight-binding lattice. The zero-energy states is sensitive to $t^{\prime}$ in the $d$ and $f$ wave symmetries because the Fermi surface lies just along the additional node lines at $k_{a}= \pm 0.25 \pi$. It is possible to consider the two types of junctions, (i.e., parallel and mirror), because of the triangular lattice structures. The Josephson effect in parallel junctions is qualitatively different that in the mirror-type junctions. We show that the Josephson current has various temperature dependences and current-phase relations depending on the pairing symmetries of superconductors, the shape of the Fermi surface and the types of the junctions.

1) D. Jerome, A. Mazaud, M. Ribault and K. Bechgaad: J. Phys. (France) Lett. 41 (1980) L92.

2) K. Bechgaad, K. Carneiro, M. Olsen, F. B. Rasmussen and C. S. Jacobsen: Phys. Rev. Lett. 46 (1981) 852.

3) I. J. Lee, M. J. Naughton, G. M. Danner and P. M. Chaikin: Phys. Rev. Lett. 78 (1997) 3555; I. J. Lee, P. M. Chaikin and M. J. Naughton: Phys. Rev. B 62 (1997) R144669.

4) I. J. Lee, S. E. Brown, W. G. Clark, M. J. Strouse, M. J. Naughton, W. Kang and P. M. Chaikin: Phys. Rev.
Lett. 88 (2002) 017004.

5) M. Takigawa, H. Yasuoka and G. Saito: J. Phys. Soc. Jpn. 56 (1987) 873.

6) S. Belin and K. Behnia: Phys. Rev. Lett. 79 (1997) 359.

7) H. I. Ha, J. I. Oh, J. Moser and M. J. Naughton: Synth. Met. 137 (2003) 1215.

8) A. A. Abrikosov: J. Low. Temp. Phys. 53 (1983) 359.

9) Y. Hasegawa and H. Fukuyama: J. Phys. Soc. Jpn. 56 (1987) 877.

10) A. G. Lebed: Phys. Rev. B 59 (1999) R721; A. G. Lebed, K. Machida and M. Ozaki: Phys. Rev. B 62 (2000) R795.

11) H. Shimahara: J. Phys. Soc. Jpn. 58 (1989) 1735.

12) K. Kuroki and H. Aoki: Phys. Rev. B 60 (1999) 3060.

13) H. Kino and H. Kontani: J. Low Temp. Phys. 117 (1999) 317.

14) K. Kuroki, R. Arita and H. Aoki: Phys. Rev. B 63 (2001) 094509.

15) J. P. Pouget and S. Ravy: J. Phys. I 6 (1996) 1501.

16) S. Kagoshima, Y. Saso, M. Maesato, R. Kondo and T. Hasegawa: Solid State Commun. 110 (1999) 479.

17) Y. Tanuma, Y. Tanaka, K. Kuroki and S. Kashiwaya: Phys. Rev. B 64 (2001) 214510.

18) Y. Tanuma, K. Kuroki Y. Tanaka, R. Arita, S. Kashiwaya and H. Aoki: Phys. Rev. B 66 (2002) 094507.

19) A. F. Andreev: Zh. Eksp. Teor. Fiz. 46 (1964) 1823. [Sov. Phys. JETP 19 (1964) 1228].

20) L. J. Buchholtz and G. Zwicknagl: Phys. Rev. B 23 (1981) 5788 .

21) C. R. Hu: Phys. Rev. Lett. 72 (1994) 1526.

22) Y. Tanaka and S. Kashiwaya: Phys. Rev. Lett. 74 (1995) 3451.

23) S. Kashiwaya and Y. Tanaka: Rep. Prog. Phys. 63 (2001) 1641.

24) T. Löfwander, V. S. Shumeiko and G. Wendin: Supercond. Sci. Technol. 14 (2001) R53.

25) Y. Asano, Y. Tanaka and S. Kashiwaya: cond-mat/0307345.

26) C. C. Tsuei and J. R. Kirtley: Rev. Mod. Phys. 72 (2000) 969.

27) D. A. Wollman, D. J. Van Harlingen, W. C. Lee, D. M. Ginsberg and A. J. Leggett: Phys. Rev. Lett. 71 (1993) 2134.

28) D. J. van Harlingen: Rev. Mod. Phys. 67 (1995) 515.

29) V. B. Geshkenbein, A. I. Larkin and A. Barone: Phys. Rev. B 36 (1987) 365.

30) M. Sigrist and T. M. Rice: J. Phys. Soc. Jpn. 61 (1992) 4283; Rev. Mod. Phys. 67 (1995) 503.

31) Y. Tanaka: Phys. Rev. Lett. 72 (1994) 3871.

32) S. Kashiwaya, Y. Tanaka, M. Koyanagi, H. Takashima and K. Kajimura: Phys. Rev. B 51 (1995) 1350.

33) S. Kashiwaya, Y. Tanaka, M. Koyanagi and K. Kajimura: Phys. Rev. B 53 (1996) 2667.

34) S. Kashiwaya, Y. Tanaka, M. Koyanagi and K. Kjimura: J. Phys. Chem. Solids 56 (1995) 1721.

35) L. Alff, H. Takashima, S. Kashiwaya, N. Terada, H. Ihara, Y. Tanaka, M. Koyanagi and K. Kajimura: Phys. Rev. B 55 (1997) 14757.

36) W. Wang, M. Yamazaki, K. Lee and I. Iguchi: Phys. Rev. B 60 (1999) 4272

37) J. Y. T. Wei, N. -C. Yeh, D. F. Garrigus and M. Strasik: Phys. Rev. Lett. 81 (1998) 2542.

38) I. Iguchi, W. Wang, M. Yamazaki, Y. Tanaka and S. Kashiwaya: Phys. Rev. B 62 (2000) R6131.

39) J. Geerk, X. X. Xi and G. Linker: Z. Phys. B 73 (1988) 329.

$40)$ Z. Q. Mao, M. M. Rosario, K. D. Nelson, K. Wu, I. G. Deac, P. Schiffer, Y. Liu, T. He, K. A. Regan and R. J. Cava: Phys. Rev. B 67 (2003) 094502.

41) J. W. Ekin, Y. Xu, S. Mao, T. Venkatesan, D. W. Face, M. Eddy and S. A. Wolf: Phys. Rev. B 56 (1997) 13746.

42) A. Sawa, S. Kashiwaya, H. Obara, H. Yamasaki, M. Koyanagi, Y. Tanaka and N. Yoshida: Physica C 339 (2000) 107.

43) H. Kashiwaya, A. Sawa, S. Kashiwaya, H. Yamazaki, M. Koyanagi, I. Kurosawa, Y. Tanaka and I. Iguchi: Physica C $357-$ 360 (2001) 1610. 
44) H. Aubin, L. H. Greene, S. Jian and D. G. Hinks: Phys. Rev. Lett. 89 (2002) 177001.

45) M. Matsumoto and H. Shiba: J. Phys. Soc. Jpn. 64 (1995) 1703.

46) Y. Nagato and K. Nagai: Phys. Rev. B 51 (1995) 16254.

47) Y. Ohashi: J. Phys. Soc. Jpn. 65 (1996) 823; Y. Ohashi and S. Takada: J. Phys. Soc. Jpn. 65 (1996) 246.

48) Y. Tanuma, Y. Tanaka, M. Yamashiro and S. Kashiwaya: Phys. Rev. B 57 (1998) 7997; Y. Tanuma, K. Kuroki Y. Tanaka and S. Kashiwaya: Phys. Rev. B 66 (2002) 174502.

49) Y. Tanaka, H. Tsuchiura, Y. Tanuma and S. Kashiwaya: J. Phys. Soc. Jpn. 71 (2002) 271; Y. Tanaka, Y. Tanuma, K. Kuroki and S. Kashiwaya: J. Phys. Soc. Jpn. 71 (2002) 2102.

50) H. X. Tang, J.-X. Zhu and Z. D. Wang: Phys. Rev. B 54 (1996) 12509; J.-X. Zhu, H. X. Tanak and Z. D. Wang: Phys. Rev. B 54 (1996) 7354.

51) N. Stefanakis: Phys. Rev. B 64 (2001) 224502; J. Phys. Cond. Matt. 13 (2001) 3643.

52) S.-T. Wu and C.-Y. Mou: Phys. Rev. B 67 (2003) 024503; Phys. Rev. B 66 (2002) 012512.

53) Z. C. Dong, D. Y. Xing and J. Dong: Phys. Rev. B 65 (2002) 214512; Z. C. Dong, D. Y. Xing, Z. D. Wang, Z. Zheng and J. Dong: Phys. Rev. B 63 (2001) 144520.

54) Yu. S. Barash, M. S. Kalenkov and J. Kurkijarvi: Phys. Rev. B 62 (2000) 6665.

55) S. Higashitani: J. Phys. Soc. Jpn. 66 (1997) 2556.

56) Y. Tanaka and S. Kashiwaya: J. Phys. Soc. Jpn. 68 (1999) 3485; J. Phys. Soc. Jpn. 69 (2000) 1152.

57) Y. Tanaka, T. Hirai, K. Kusakabe and S. Kashiwaya: Phys. Rev. B 60 (1999) 6308; T. Hirai, K. Kusakabe and Y. Tanaka: Physica C 336 (2000) 107; K. Kusakabe and Y. Tanaka: Physica C 367 (2002) 123; K. Kusakabe and Y. Tanaka: J. Phys. Chem. Solids 63 (2002) 1511.

58) C. Honerkamp and M. Sigrist: J. Low. Temp. Phys. 111 (1998) 898; Prog. Theor. Phys. 100 (1998) 53.

59) K. Sengupta, I. Žutić, H.-J. Kwon, V. M. Yakovenko and S. Das Sarma: Phys. Rev. B 63 (2001) 144531.

60) S. Shirai, H. Tsuchiura, Y. Asano, Y. Tanaka, J. Inoue, Y. Tanuma and S. Kashiwaya: J. Phys. Soc. Jpn. 72 (2003) 2299.

61) H. Tsuchiura, Y. Tanaka and Y. Ushijima: J. Phys. Soc. Jpn 64 (1995) 922.

62) M. Yamashiro, Y. Tanaka and S. Kashiwaya: Phys. Rev. B 56 (1997) 7847.

63) M. Yamashiro, Y. Tanaka, Y. Tanuma and S. Kashiwaya: J. Phys. Soc. Jpn. 67 (1998) 3224.

64) M. Yamashiro, Y. Tanaka, N. Yoshida and S. Kashiwaya: J. Phys. Soc. Jpn. 68 (1999) 2019.

65) Yu. S. Barash, A. M. Bobkov and M. Fogelstrom: Phys. Rev. B 64 (2001) 214503.

66) Y. Asano and K. Katabuchi: J. Phys. Soc. Jpn. 71 (2002) 1974.

67) Y. Asano, Y. Tanaka, M. Sigrist and S. Kashiwaya: Phys. Rev. B 67 (2003) 184505.

68) Y. Asano, Y. Tanaka, Y. Matsuda and S. Kashiwaya: condmat/0306155.

69) J-X. Zhu, B. Friedman and C. S. Ting: Phys. Rev. B 59 (1999) 9558.

70) S. Kashiwaya, Y. Tanaka, N. Yoshida and M. R. Beasley: Phys. Rev. B 60 (1999) 3572.

71) I. Zutic and O. T. Valls: Phys. Rev. B 60 (1999) 6320.

$72)$ N. Yoshida, Y. Tanaka, J. Inoue and S. Kashiwaya: J. Phys. Soc. Jpn. 68 (1999) 1071.

73) N. Yoshida, H. Itoh, T. Hirai, Y. Tanaka, J. Inoue and S. Kashiwaya: Phsica C 367 (2002) 135.

74) N. Yoshida, Y. Asano, H. Itoh, Y. Tanaka and J. Inoue: J. Phys. Soc. Jpn. 72 (2003) 895.

75) T. Hirai, N. Yoshida, Y. Tanaka, J. Inoue and S. Kashiwaya: J. Phys. Soc. Jpn. 70 (2001) 1885.

76) T. Hirai, Y. Tanaka, N. Yoshida, Y. Asano, J. Inoue and S. Kashiwaya, Phys. Rev. B 67 (2003) 174501.

77) M. Fogelström, D. Rainer and J. A. Sauls: Phys. Rev. Lett.
79 (1997) 281.

78) M. Covington, M. Aprili, E. Paraoanu, L. H. Greene, F. Xu, J. Zhu and C. A. Mirkin: Phys. Rev. Lett. 79 (1997) 277.

79) A. Biswas, P. Fournier, M. M. Qazilbash, V. N. Smolyaninova, H. Balci and R. L. Greene: Phys. Rev. Lett. 88 (2002) 207004 .

80) Y. Dagan and G. Deutscher: Phys. Rev. Lett. 87 (2001) 177004 .

81) A. Sharoni, O. Millo, A. Kohen, Y. Dagan, R. Beck, G. Deutscher and G. Koren: Phys. Rev. B 65 (2002) 134526.

82) A. Kohen, G. Leibovitch and G. Deutscher: Phys. Rev. Lett. 90 (2003) 207005.

83) M. Matsumoto and H. Shiba: J. Phys. Soc. Jpn. 64 (1995) 4867.

84) R. B. Laughlin: Phys. Rev. Lett. 80 (1998) 5188.

85) I. Lubimova and G. Koren: cond-mat/0306030.

86) N. Kitaura, H. Itoh, Y. Asano, Y. Tanaka, J. Inoue, Y. Tanuma and S. Kashiwaya: J. Phys. Soc. Jpn. 72 (2003) 1718.

87) Y. Tanuma, Y. Tanaka, M. Ogata and S. Kashiwaya: J. Phys. Soc. Jpn. 67 (1998) 1118.

88) Y. Tanuma, Y. Tanaka, M. Ogata and S. Kashiwaya: Phys. Rev. B 60 (1999) 9817.

89) Y. Tanuma, Y. Tanaka and S. Kashiwaya: Phys. Rev. B 64 (2001) 214519.

90) M. M. Qazilbash, A. Biswas, Y. Dagan, R. A. Ott and R. L. Greene: Phys. Rev. B 68 (2003) 024502.

91) L. Alff, S. Kleefishch, U. Schoop, M. Zittartz, T. Kemen, T. A. Marx and R. Gross: Eur. Phys. J. B 5 (1998) 423.

92) A. Sawa, S. Kashiwaya, H. Kashiwaya, H. Obara, H. Yamasaki, M. Koyanagi, I. Kurosawa and Y. Tanaka: Physica C 357-360 (2001) 294.

93) Y. Tanaka, H. Itoh, H. Tsuchiura, Y. Tanuma, J. Inoue and S. Kashiwya: J. Phys. Soc. Jpn. 71 (2002) 2005.

94) Y. S. Barash, A. A. Svidzinsky and H. Burkhardt: Phys. Rev. B 55 (1997) 15282.

95) A. A. Golubov and M. Y. Kupriyanov: Pis'ma Zh. Eksp. Teor. fiz 69 (1999) 242.[ Sov. Phys. JETP Lett. 69 (1999) 262.]; 67 (1998) 478.[ Sov. Phys. JETP Lett. 67 (1998) 501.]

96) A. Poenicke, Yu. S. Barash, C. Bruder and V. Istyukov: Phys. Rev. B 59 (1999) 7102.

97) K. Yamada, Y. Nagato, S. Higashitani and K. Nagai: J. Phys. Soc. Jpn. 65 (1996) 1540.

98) Y. Tanaka, Y. Tanuma and S. Kashiwaya: Phys. Rev. B 64 (2001) 054510.

99) T. Lück, U. Eckern and A. Shelankov: Phys. Rev. B 63 (2001) 064510.

100) Y. Tanaka, T. Asai, N. Yoshida, J. Inoue and S. Kashiwaya: Phys. Rev. B 61 (2000) R11902.

101) Y. Asano and Y. Tanaka: Phys. Rev. B 65 (2002) 064522; "Toward the controllable Quantum State" Eds. H. Takayanagi and J. Nitta, 185, (World Scientific, Singapole, 2003).

102) Y. Asano, Y. Tanaka and S. Kashiwaya: cond-mat/0302287.

103) Y. Asano: Phys. Rev. B 64 (2001) 014511.

104) Y. Asano: J. Phys. Soc. Jpn. 71 (2002) 905; Y. Asano: Physica C 367 (2002) 92; Y. Asano: Physica C 367 (2002) 157.

105) Y. Tanaka, Yu. V. Nazarov and S. Kashiwaya: Phys. Rev. Lett. 90 (2003) 167003.

106) Y. S. Barash, H. Burkhardt and D. Rainer: Phys. Rev. Lett. $\mathbf{7 7}$ (1996) 4070.

107) Y. Tanaka and S. Kashiwaya: Phys. Rev. B 53 (1996) 9371.

108) Y. Tanaka and S. Kashiwaya: Phys. Rev. B 53 (1996) R11957.

109) Y. Tanaka and S. Kashiwaya: Phys. Rev. B 56 (1997) 892.

110) Y. Tanaka and S. Kashiwaya: Phys. Rev. B 58 (1998) R2948.

111) Y. Asano: Phys. Rev. B 64 (2001) 224515.

112) R. A. Riedel and P. F. Bagwell: Phys. Rev. B 57 (1998) 6084.

113) M. P. Samanta and S. Datta: Phys. Rev. B 55 (1997) R8689.

114) N. Stefanakis: Phys. Rev. B 65 (2002) 064533.

115) E. Il'ichev, V. Zakosarenko, R. P. IJsselsteijn, H.-G. Meyer and H. E. Hoenig: Phys. Rev. Lett. 81 (1998) 894.

116) E. Ilichev, M. Grajcar, R. Hlubina, R. P. IJsselsteijn, H. E. Hoenig, H.-G. Meyer, A. Golubov, M. H. S. Amin, 
A. M. Zagoskin, A. N. Omelyanchouk and M. Yu. Kuprianov: Phys. Rev. Lett. 86 (2001) 53.

117) H. Arie, K. Yasuda, H. Kobayashi and I. Iguchi: Phys. Rev. B 62 (2000) 11864.

118) H. Hilgenkamp, J. Mannhart and B. Mayer: Phys. Rev. B 53 (1996) 14586.

119) F. Lonbardi, F. Tafuri, F. Ricci, F. Miletto Granozio, A. Barone, G. Testa, E. Sarnelli, J. R. Kirtley and C. C. Tsuei: Phys. Rev. Lett. 89 (2002) 207001.

120) H. J. H. Smilde, Ariando, D. H. A. Blanko, G. J. Gerritsma, H. Hilgenkamp and H. Rogalla: Phys. Rev. Lett. 88 (2002) 057004 .

121) H. Hilgenkamp and J. Mannhart: Rev. Mod. Phys. 74 (2002) 485.
122) T. Imaizumi, T. Kawai, T. Uchiyama and I. Iguchi: Phys. Rev. Lett. 89 (2002) 017005.

123) C. D. Vaccarella, R. D. Duncan and C. A. R. Sa de Melo: Physica C 391 (2003) 89.

124) H.-J. Kwon, K. Senguputa and V. M. Yakovenko: condmat/0210148.

125) P. G. de Gennes: Superconductivity of Metals and Alloys, (Benjamin, New York, 1966).

126) P. A. Lee and D. S. Fisher: Phys. Rev. Lett. 47 (1981) 882.

127) A. Furusaki: Physica B. 203 (1994) 214.

128) Y. Asano: Phys. Rev. B 63 (2001) 052512.

129) Y. Asano: Phys. Rev. B 66 (2002) 174506.

130) V. Ambegaokar and A. Baratoff: Phys. Rev. Lett. 10 (1963) 486 